\title{
Comparison of macroscopic descriptions of magnetization curves
}

\author{
Radosław Jastrzębski ${ }^{1,{ }^{*}}$, and Krzysztof Chwastek ${ }^{1}$ \\ ${ }^{1}$ Częstochowa University of Technology, Faculty of Electrical Engineering, 42-201 Częstochowa, Poland
}

\begin{abstract}
The paper considers a qualitatively different behaviour of two phenomenological hysteresis models. The first one is the widespread Jiles-Atherton description, which is based on the "effective field" concept. The other model is the proposal by the Brazilian research team GRUCAD. First order reversal curves simulated with the latter formalism do not exhibit negative slopes. This feature is in accordance with the experiment.
\end{abstract}

\section{Introduction}

Among different descriptions of magnetization curves [1] the phenomenological Jiles-Atherton (JA) model $[2,3]$ has attracted a lot of attention of the scientific and engineering community due to a relatively simple mathematical form and physical interpretation attributed to its few parameters. However, soon after the JA model gained popularity, many researchers have come to the conclusion that this description has a number of drawbacks. The original iterative estimation procedure proposed by Jiles et al. [4] sometimes gave nonconsistent sets of model parameters [5, 6]. Therefore most of the research focused on alternative methods for estimation of model parameters, and was usually based on artificial intelligence approaches [5-16]. At the same time several authors realized that in order to obtain a reliable representation of more complex magnetization cycles, including minor loops, reversal curves etc. it would be necessary to update the values of model parameters in accordance with certain functional dependencies $[6,10,17-20]$. The abundance of different model versions and the focus set mostly on the advantages of one estimation method over another have blurred serious problems with the model equations themselves.

The model developed by researchers from a Brazilian group GRUCAD [21-23] was originally meant as a ,patch" to the JA description. In our paper we shall show that the introduced modifications introduce a new quality.

\section{JA model equations}

The fundamental ideas behind the JA model are the concept of anhysteretic curve and the effective field. Anhysteretic curve is the purely reversible $M-H$ dependence. Jiles and Atherton suggested for its description the use of the so-called modified Langevin curve, given with Eq. (1)

$$
M_{\text {an }}=M_{\mathrm{s}}\left(\operatorname{coth} \frac{H_{e f f}}{a}-\frac{a}{H_{e f f}}\right),
$$

where $a(\mathrm{~A} / \mathrm{m})$ and $M_{\mathrm{s}}(\mathrm{A} / \mathrm{m})$ are model parameters, whereas $H_{\text {eff }}$ is the so-called effective field interpreted as the true field with the magnetic material, different from the applied field. The specification "modified" in the name of the curve is to focus the attention of the user on this fact (ordinary Langevin function uses the applied field $H$ as its argument). The effective field is given in the first approximation with the relationship

$$
H_{\text {eff }}=H+\alpha M \text {, }
$$

where dimensionless $\alpha$ is yet another model parameter, describing the mutual interactions between magnetic moments within the material. It should be recalled that the concept of effective field enables incorporating in the description the cooperative contributions from different physical phenomena affecting the shape of hysteresis loop i.e. $H_{e f f}=H+\alpha M+\sum_{i} H_{i}$, thus a generalized model in the self-consistent form $M=M\left(H_{e f f}\right)$ [24] may easily be created. For example Sablik and Jiles [25] suggested introducing an additional field term related to magneto-mechanical coupling; this model extension has attracted a lot of attention of researchers concerned with non-destructive testing methods $[26,27]$. The idea of effective field may also be helpful when explaining the peculiarities of magnetization process in novel soft magnetic materials like Fe-based composites [28In general, the concept allows for qualitative and quantitative description of the effects from complicated factors and phenomena, which would otherwise be very difficult to analyze.

\footnotetext{
* Corresponding author: radoslaw@jastrzebski.info
} 
The fundamental equation of the JA model relates the derivative of total magnetization with respect to the effective field to the weighted difference between anhysteretic and total magnetizations, which may be written as an ordinary differential equation (ODE)

$$
\frac{\mathrm{d} M}{\mathrm{~d} H_{\text {eff }}}=\frac{M_{\mathrm{an}}-M}{k \delta},
$$

where $\delta= \pm 1$ is introduced to distinguish the ascending and the descending hysteresis loop branches, whereas $k$ is a model parameter (if expressed in $(\mathrm{A} / \mathrm{m})$ taking values comparable to quasi-static coercive field strength). In certain versions of the model instead of total magnetization $M$, its component related to irreversible magnetization processes is used. These modifications do not alter significantly the logical derivation of the final formula or the qualitative model behaviour. A step-bystep derivation is provided e.g. in [29-30], whereas the differences between subsequent versions and their consequences are discussed in [31].

As pointed out in the previous paragraph, the model developers attempted to decompose total magnetization into its reversible and irreversible components. The original paper [2] did not include this idea, however most cited papers on the JA model, [3] and [4], offered such a possibility. The formulas in both papers showed slight differences, yet it can be stated that the decomposition was performed by weighting the appropriate contributions with a new dimensionless parameter $\mathrm{c}$. The interpretation of this parameter was the ratio of differential susceptibility $\mathrm{d} M / \mathrm{d} H$ to differential anhysteretic susceptibility $\mathrm{d} M_{\text {an }} / \mathrm{d} H$ at the origin of the coordinate system. At this point it should be remarked that the Langevin function has a singularity at zero, which makes the parameter imprecisely defined. The Maclaurin expansion only partially resolves the problem. The value of the $c$ parameter is subject to unexpected, sudden variations if a stochastic optimization routine applied for solving the estimation problem is repeated several times to ensure convergence [32].

Another paper [33] criticized the approach based on decomposition of total magnetization, considering it artificial and borrowed from the Preisach model [34]. In order to improve the representation of minor loops including asymmetric ones, Chwastek introduced a decomposition of total susceptibility into two terms, but both were modulated with an instant magnetization value, thus the modified JA description [20] resembled to a certain extent the product of the Preisach model [34]. It was, however, necessary to update the values of two model parameters depending on the magnetization level.

Yet another modification had to be introduced, namely the introduction of additional pseudo-variable $\delta_{\mathrm{M}}$ in front of the difference $M_{\text {an }}-M_{\text {irr }}$, which appears in an equation similar to Eq. (3). The definition of $\delta_{\mathrm{M}}$ is given below:

$$
\delta_{\mathrm{M}}=\left\{\begin{array}{c}
0: \mathrm{d} H / \mathrm{d} t<0 \text { and } M_{\text {an }}-M_{\text {irr }}>0 \\
0: \mathrm{d} H / \mathrm{d} t>0 \text { and } M_{\text {an }}-M_{\mathrm{irr}}<0 \\
1: \text { in all other cases }
\end{array},\right.
$$

or in a more brief notation:

$$
\delta_{\mathrm{M}}=0.5\left[1+\operatorname{sign}\left(\left(M_{\mathrm{an}}-M_{\mathrm{irr}}\right) \mathrm{d} H / \mathrm{d} t\right)\right] .
$$

In the relationships (4) and (5) there might be slight modifications relative to the model version, namely the difference might be $M_{\text {an }}-M$ and $\mathrm{d} H / \mathrm{d} t$ may be replaced with $\mathrm{d} B / \mathrm{d} t$ for the inverse model [20, 35]. The meaning of $\delta_{\mathrm{M}}$ pseudo-variable may be inferred from Fig. 1. Its role is to suppress the irreversible magnetization component after a sudden field reversal. Such a modification may be partially justified with the words by E. Della Torre et al: "In general, the slope of the $M-H$ curve is different before and after a turning point. The reason for the difference in slope is that before the turning point, the change in magnetization is due to both the reversible and the irreversible processes. After the turning point, only the reversible process contributes. This is due to the fact that the irreversible process does not return any energy to the applied field; hence, $\mathrm{d} M / \mathrm{d} H$ is zero for this process" [36].

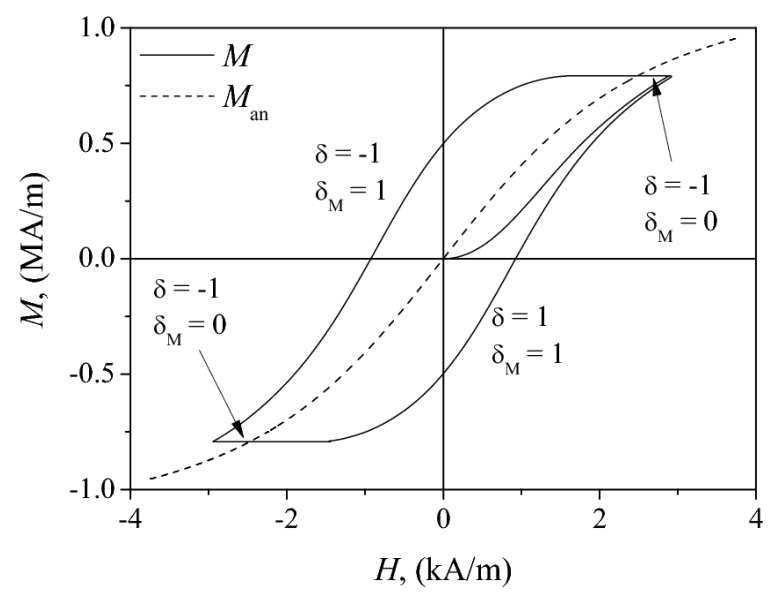

Fig. 1. A hysteresis loop and an anhysteretic curve simulated with the JA model that includes $\delta_{\mathrm{M}}$ term.

The necessity to patch JA model equations with the $\delta_{\mathrm{M}}$ pseudo-variable described above was mentioned for the first time in the paper on estimation [3] and explicitly appeared in Ref. [37]. However, it cannot be traced back to any ideas inherent in the model and thus should be considered as an artificial means to patch the JA equations. Without this pseudovariable, after a sudden change of the sign of the magnetic field a loop fragment with a negative differential susceptibility would be observed. This could lead to the instability of FEM codes when calculating the local field distribution taking into account the hysteresis of the medium $[38,39]$. 


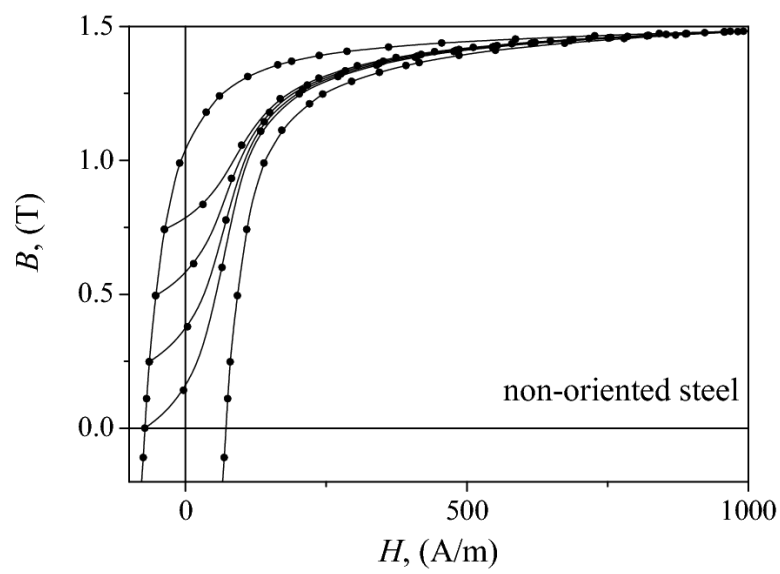

Fig. 2. Experimental first order reversal curves for a nonoriented steel measured using Epstein frame [40].

Experiments were carried out on custom- designed laboratory set-ups, enabled introducing bias signal to the excitation, thus allowing us to obtain asymmetric minor loops and reversal curves indicate unambiguously that after sudden field reversal the slope of the $M-H$ curve is non-zero, cf. Fig. 2. Therefore, the introduction of $\delta_{\mathrm{M}}$ pseudo-variable is not fully justified. The term has been introduced post hoc to the quasi-static model equations to remove the undesirable model behaviour and its existence is in a strict connection to the form of the right hand side expression in (3), whose interpretation may be that the instant value of magnetization $M(t)$ on the hysteresis loop is obtained by introducing an appropriate offset from the instant value of anhysteretic magnetization $M_{\text {an }}(t)$.

Yet another interesting problem concerns the following point: both quantities are implicitly related by time (they should be calculated for the same time instants). We are therefore faced with two possibilities: either the anhysteretic (hysteresis-free) curve in reality does exhibit hysteresis if we follow rigorously all definitions, or that it should be calculated from a selfconsistent implicit relationship similar to (1), as suggested e.g. in Refs. [41, 42]

$$
M_{\mathrm{an}}=M_{\mathrm{s}}\left(\operatorname{coth} \frac{H+\alpha M_{\mathrm{an}}}{a}-\frac{a}{H+\alpha M_{\mathrm{an}}}\right) .
$$

It should be noted that in the latter case the ,effective field" has been implicitly redefined, which may have had consequences for the derivation of the final formula for differential susceptibility. Moreover, for certain sets of model parameters $M_{\mathrm{s}}, \alpha$ and $a$ the curve (6) may become S-slanted and go into the second and fourth quadrant of the $M-H$ plane, which in turn implies the non-physical shape of the hysteresis loop or failure of the estimation procedure $[33,39]$. More information on the issue may be found in the Appendix.

Considering the first of the aforementioned cases, i.e. all definitions of respective quantities are rigorously followed, it is instructive to follow the logical scheme of calculations for the case of the so-called inverse model, i.e. when in the calculations the input quantity is magnetic flux density $B \quad[30,35,43]$. The toned for using the inverse model stems from the fact that in magnetic measurements, carried out in accordance with international standards IEC 60404, the shape of magnetic flux density waveform should be kept sinusoidal. Three quantities $B, M$ and $H$ are related to each other with the constitutive relationship $B=\mu_{0}(H+M)$, where $\mu_{0}$ is free space permeability. Now treating $B(t)$ as the input quantity to the inverse model, the calculation sequence is as follows:

1. define $B_{\max }$

2. set up initial values $M(t=0) \cong 0, H(t=0) \cong 0$

3. calculate $H_{\mathrm{eff}}(t)=H(t)+\alpha M(t)$

4. calculate $M_{\text {an }}(t)=M_{s}\left(\operatorname{coth} H_{\text {eff }}(t) / a-a / H_{\text {eff }}(t)\right)$

5. calculate instant value of $\mathrm{d} M / \mathrm{d} B$ and for the prescribed $\mathrm{d} B / \mathrm{d} t$ waveform calculate $\mathrm{d} M / \mathrm{d} t$

6. integrate $\mathrm{d} M / \mathrm{d} t$, thus determine the instant value of $M(t+\Delta t)$

7. calculate the value of field strength for the successive time instant $H(t+\Delta t)$ from the transformer constitutive relationship $H(t+\Delta t)=B(t+\Delta t) / \mu_{0}-M(t+\Delta t)$

8. assume next time step and go to step 3 .

Integration of model equations may be carried out using standard library Runge-Kutta routines. After several time steps the vectors $B(t), M(t)$ and $H(t)$ are created in computer memory.

Next the vectors $H_{\text {eff }}(t)=H(t)+\alpha M(t)$ and $M_{\text {an }}(t)=M_{\mathrm{s}}\left[\operatorname{coth} H_{\text {eff }}(t) / a-a / H_{\text {eff }}(t)\right]$ are set up. The anhysteretic magnetization $M_{\text {an }}(t)$ may be represented on the $M-H$ plane as in Fig. 1, where it took the sigmoid shape, as in fact the dependence $M_{\text {an }}=M_{\text {an }}\left(H_{\text {eff }}\right)$ was depicted with a broken line, or as in Fig. 3, where the dependence $M_{\text {an }}=M_{\text {an }}(H)$ is drawn. For creating both figures the same set of model parameters was used.

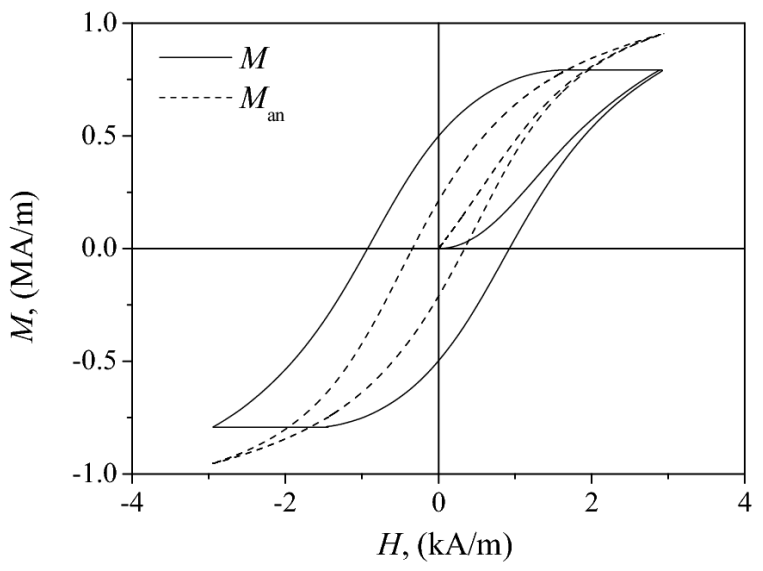

Fig. 3. The ,anhysteretic" curve given with the modified Langevin function in fact exhibits hysteresis [42]

Summing up to this point, the following remarks on the original JA description may be made: 
- it is based on the concept of the effective field, which appears explicitly in the fundamental model equation (3) and the modified Langevin function (1) as the independent variable;

- the loop branches are constructed in the description by the introduction of an offset from the ,,anhysteretic" curve (1);

- the direct introduction of the effective field as the argument to the ,anhysteretic" curve may lead to serious interpretation problems;

- it is necessary to ,,patch" quasi-static model equations using the $\delta_{\mathrm{M}}$ pseudo-variable in order to get rid of the regions with negative differential susceptibilities; this introduces characteristic flat fragments of the simulated curves;

- the proposed modification only partially resolves the problem; in fact this is an artificial solution. For a hysteresis description devoid of the global memory mechanism, such as the JA model [44], it is always possible to perform a coordinate transformation by moving the reversal point to the origin. After such transformation the slope of the $M-H$ curve (the differential susceptibility) should be positive and its value should correspond to the Rayleigh relationship.

\section{GRUCAD model equations}

In Refs. [21-23] the researchers from the Brazilian team GRUCAD introduced an alternative description. A distinct feature of the model is that the reversible and irreversible processes are separated from each other. Moreover their summing is carried out along the $H$ axis. In the present paper we consider the set of equations, as outlined in Refs. [45, 46].

The reversible (anhysteretic) part is described with two equations:

$$
\begin{gathered}
H_{\mathrm{an}}=B / \mu_{0}-M_{\mathrm{s}}(\operatorname{coth} \lambda-1 / \lambda), \\
\lambda=\frac{1}{a}\left[(1-\alpha) H_{\mathrm{an}}+\alpha \frac{B}{\mu_{0}}\right] .
\end{gathered}
$$

The parameter $\alpha$ plays the role of a weighting coefficient between the flux density $B$ and the reversible field strength $H_{\mathrm{an}}$. The parameter $a$ adjusts the level of $\lambda$ for the Langevin function in (7).

$$
\frac{\mathrm{d} H_{\mathrm{h}}}{\mathrm{d} B}=\frac{H_{\mathrm{Hs}}\left(\operatorname{coth} \lambda_{\mathrm{H}}-1 / \lambda_{\mathrm{H}}\right)+H_{\mathrm{h}}}{\gamma \delta}
$$

with $\lambda_{\mathrm{H}}$ defined as

$$
\lambda_{\mathrm{H}}=\frac{H_{\mathrm{h}}+\delta H_{\mathrm{HS}}}{a}
$$

where $\delta= \pm 1$ is the sign of $\mathrm{d} B / \mathrm{d} t$.
Equations (9) and (10) constitute the description of irreversible (hysteretic) part. It is given in the form of an ordinary differential equation whose independent variable is the flux density $B$. Thus the GRUCAD proposal is inherently an inverse ( $B$ - input ) model. Such models are useful in two dimensional FEM analyses of magnetic circuits, where the magnetic vector potential A may be introduced $(\mathbf{B}=\operatorname{curl} \mathbf{A})^{\mathrm{a}}$.

Finally the reversible and irreversible contributions are described by

$$
H=H_{\text {an }}+H_{\mathrm{h}}
$$

The model parameters are $\alpha, a, \gamma, H_{\mathrm{HS}}$ and $M_{\mathrm{s}}$. The effects of their variations on the shape of hysteresis loop is discussed in [45]. The physical meaning of parameters $\alpha$ and $a$ is altered when compared to the JA model. The first one no longer represents the cooperative effects resulting from the presence of ,effective field"- the concept of effective field is simply abandoned. Finally the relationship (11) deserves attention. The branches of hysteresis loop are obtained by introducing appropriate offsets from the truly anhysteretic curve along the $H$ axis and not along the $M$ axis, as in the original JA model. Therefore, there is no need to patch the model equations using the inconvenient $\delta_{\mathrm{M}}$ pseudo-variable. The model is somewhat similar in spirit to other descriptions consistent with the laws of irreversible thermodynamics, like those discussed in Refs. [4749].

Figure 4 depicts a saturating hysteresis loop and some first order reversal curves (FORCs) simulated with the GRUCAD model. The model parameters were specified after [45], namely: $\alpha=9.48 \cdot 10^{-5}(-)$, $a=40(\mathrm{~A} / \mathrm{m}), M_{\mathrm{s}}=1.2 \cdot 10^{6}(\mathrm{~A} / \mathrm{m}), \gamma=0.12(\mathrm{~A} / \mathrm{m})$ and $H_{\mathrm{HS}}=50(\mathrm{~A} / \mathrm{m})$. These parameters may describe well a sample of non-oriented electrical steel. The FORCs were chosen to start on the descending branch of the major loop. A precise choice of their beginning may be controlled in Matlab using the events feature available for the ODE solvers. In the inset a zoomed fragment of the $B-H$ plane is shown. It is evident that the FORCs do not exhibit negative slopes. Moreover, it can be stated that the first FORC almost overlaps the virgin curve, which is depicted with a broken line in the inset (the initial conditions for ODE were set to illustrate the resemblance between these two curves). The similarity of the pattern exhibited by magnetization curves was noticed more than a century ago [50]. It was practically implemented in a simple phenomenological hysteresis model $[51,52]$.

a A standard quasi-static $H$ - input hysteresis model like the original Jiles-Atherton model may always be transformed to the inverse form using the identity $\frac{\mathrm{d} M}{\mathrm{~d} H}=\frac{\mathrm{d} M}{\mathrm{~d} B} \frac{\mathrm{d} B}{\mathrm{~d} H}=\frac{\mathrm{d} M}{\mathrm{~d} B} \mu_{0}\left(1+\frac{\mathrm{d} M}{\mathrm{~d} H}\right)$ 


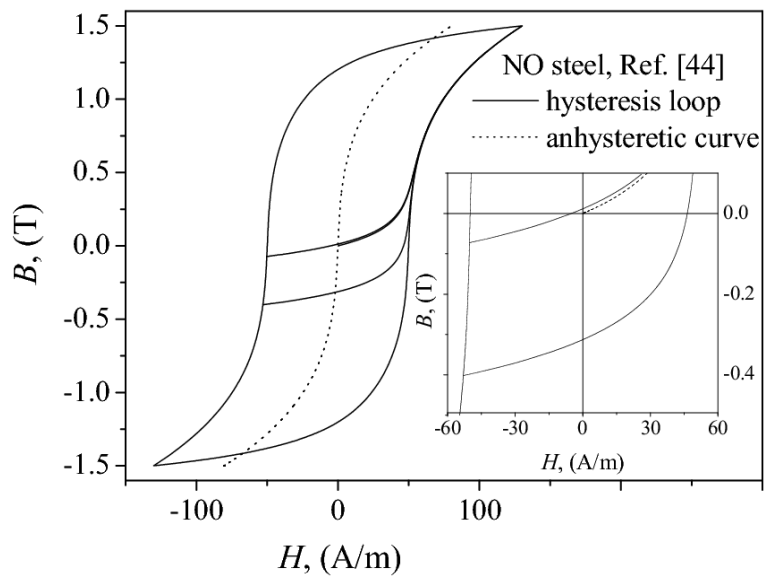

Fig. 4. The hysteresis loop at $B_{\mathrm{m}}=1.5 \mathrm{~T}$ and two FORCs simulated with the GRUCAD model.

\section{Conclusions}

In the paper an analysis of two phenomenological hysteresis models was carried out. First of them was the widespread Jiles-Atherton proposal, and the other one a description advanced by the Brazilian research team GRUCAD. Attention was focused on qualitatively different behaviour of the models after sudden field reversal. First order reversal curves simulated with the GRUCAD description do not exhibit negative slopes, which makes this model useful for implementation in FEM codes.

\section{Appendix}

Recently Padilla et al. published a paper [39], which focused on the problem of negative slopes of hysteresis loops modelled with the JA model for specified sets of parameters. In the Appendix we apply the analytical approximation to the inverse Langevin function to illustrate the issue for the anhysteretic curve given with expression (6).

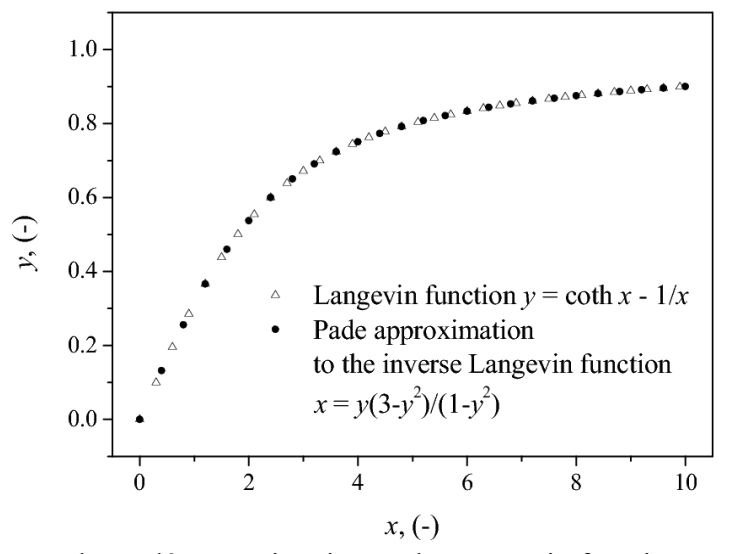

Fig. 5. The Padé approximation to the Langevin function.

The Padé approximation [53, 54] yields a simple formula for the inverse of the Langevin function

$$
\text { if } y=\operatorname{coth} x-1 / x \text { then } x=y \frac{3-y^{2}}{1-y^{2}}
$$

As it can be inferred from Fig. 5, the approximation offers results acceptable for engineering purposes.

Applying the considered approximation to (6) we obtain a relationship that may be solved for $H$ :

$$
H+\alpha M_{\mathrm{an}}=\frac{a M_{\mathrm{an}}}{M_{\mathrm{s}}}\left(\frac{3-\left(\frac{M_{\mathrm{an}}}{M_{\mathrm{s}}}\right)^{2}}{1-\left(\frac{M_{\mathrm{an}}}{M_{\mathrm{s}}}\right)^{2}}\right)
$$

The critical set of model parameter shall occur when the derivative $\mathrm{d} H /\left.\mathrm{d} M_{\text {an }}\right|_{M_{\text {an }} \rightarrow 0}$ takes the zero value. It means the following relationship between $\alpha, a$ and $M_{\mathrm{s}}$ :

$$
\alpha_{\text {crit }}=\frac{3 a_{\text {crit }}}{M_{\mathrm{s}}}
$$

A Similar derivation may be carried out for the GRUCAD model parameters.

\section{References}

1. A. Iványi, Hysteresis models in electromagnetic computation (Akadémiai Kiadó, Budapest 1997)

2. D. C. Jiles, D. L. Atherton, IEEE Trans. Magn. 19, 2183 (1983)

3. D. C. Jiles, D. L. Atherton, J. Magn. Magn. Mater. 61, 48 (1986)

4. D. C. Jiles, J. B. Thoelke, M. K. Devine, IEEE Trans. Magn. 28, 27 (1992)

5. D. A. Philips, L. R. Dupré, J. A. Melkebeek, IEEE Trans. Magn. 31, 3551 (1995)

6. D. Lederer, H. Igarashi, A. Kost, T. Honma, IEEE Trans. Magn. 35, 1211 (1999)

7. P. R. Wilson, J. Neil Ross, A. D. Brown, IEEE Trans. Magn. 38, 1049 (2001)

8. A. Salvini, F. Riganti Fulginei, IEEE Trans. Magn. 41, 1100 (2005)

9. K. Chwastek, J. Szczygłowski, J. Magn. Magn. Mater. 314, 47 (2007)

10. R. Szewczyk, J. Phys. D : Appl. Phys. 40, 4109 (2007)

11. R. Marion, R. Scoretti, N. Siauve, M.-A. Raulet, IEEE Trans. Magn. 44, 894 (2008)

12. M. Toman, G. Štumberger, D. Dolinar, IEEE Trans. Magn. 44, 1098 (2008)

13. A. P. S. Baghel, S. V. Kulkarni, IET Electr. Power Appl. 6, 689 (2012)

14. R.-A. Naghizadeh, B. Vahidi, S.H. Hosseinian, COMPEL 31, 1293 (2012)

15. Lu Hai-Liang, Wen Xi-Shan, Lan Lei, An YunZhu, Li Xiao-Ping, J. Magn. Magn. Mater. 374, 502 (2015) 
16. M. A. Zaman, P. C. Hansen, L. T. Neustock, P. Padhy, L. Hesselink, J. Appl. Phys. 120, 093903 (2016)

17. Z. Włodarski, IEEE Trans. Magn. 39, 1990 (2003)

18. A. Benabou, J. V. Leite, S. Clénet, C. Simão, N. Sadowski, J. Magn. Magn. Mater. 320, e1034 (2008)

19. K. Chwastek, J. Phys. D : Appl. Phys. 42, 165002 (2009)

20. Zb. Gmyrek, Int. J. Numer. Model. 27, 199 (2014)

21. P. I. Koltermann, L. A. Righi, J. P. A. Bastos, R. Carlson, N. Sadowski, N. J. Batistela, Physica B 275, 233 (2000).

22. L. A. Righi, N. Sadowski, R. Carlson, J. P. A. Bastos, N. J. Batistela, IEEE Trans. Magn. 37, 3353 (2001)

23. P. I. Koltermann, J. P. A. Bastos, N. Sadowski, N. J. Batistela, A. Kost, L. Jänicke, K. Miethner, IEEE Trans. Magn. 38, 897 (2002)

24. P. Andrei, A. Stancu, H. Hauser, P. Fulmek, J. Optoelectr. Adv. Mater. 9, 1137 (2007)

25. M. Sablik, D. C. Jiles, IEEE Trans. Magn. 29, 2113 (1993)

26. K. J. Stevens, NDT\&E 33, 111 (2000)

27. Jianwei Li, Minqiang $\mathrm{Xu}$, J. Appl. Phys. 110, 063918 (2011)

28. A. Jakubas, P. Gębara, S. Seme, A. Gnatowski, K. Chwastek, Acta Phys. Pol. A 131, 1289 (2017)

29. R. Venkataraman, P. S. Krishnaprasad, Proc. 37th IEEE Conf. Decision and Control, Tampa, USA, 1998, doi:10.1109/CDC.1998.757777

30. N. Sadowski, N. J. Batistela, J. P. A. Bastos, M. Lajoie-Mazenc, IEEE Trans. Magn. 38, 797 (2002)

31. K. Chwastek, J. Szczygłowski, Przegl. Elektrotechn. 12, 145 (2008)

32. R. Marion, N. Siauve, M.-A. Raulet, L. Krähenbühl, K. Chwastek, J. Szczygłowski, W. Wilczyński, A comparison of identification techniques for the Jiles-Atherton model of hysteresis, presented at XX EPNC 2-4.07.2008, Lille, France
33. S. E. Zirka, Yu. I. Moroz, R. G. Harrison, K. Chwastek, J. Appl. Phys. 112, 043916 (2012)

34. I. D. Mayergoyz, Mathematical models of hysteresis, Springer, New York, 1991

35. B. Vaseghi, D. Mathekga, S. Rahman, A. M. Knight, IEEE Trans. Magn. 49, 1637 (2013)

36. E. Della Torre, J. Oti, G. Kadár, IEEE Trans. Magn. 26, 3052 (1990)

37. J. H. B. Deane, IEEE Trans. Magn. 30, 2795 (1994)

38. H. Toms, R. Colclaser, M. Krefta, IEEE Trans. Magn. 2, 982 (2008)

39. J. B. Padilha, P. Kuo-Peng, N. Sadowski, J. V. Leite, N. J. Batistela, J. Magn. Magn. Mater. 442, 8 (2017)

40. http://www.zirka.dp.ua

41. D. C. Jiles, J. Appl. Phys. 76, 5849 (1994)

42. Liu Qingyou, Luo Xu, Zhu Haiyan, Liu Jianxun, Han Yiwei, Chinese Phys. B 26 (7), 077502 (2017)

43. K. Chwastek, Math. Comput. Model. Dyn. Syst. 15, 95 (2009)

44. T. Kleineberg, J. Magn. Magn. Mater. 166, 315 (1997)

45. S. Steentjes, K. Chwastek, M. Petrun, D. Dolinar, K. Hameyer, IEEE Trans. Magn. 50, 7300804 (2014), doi: 10.1109/TMAG.2014.2323250

46. R. Jastrzębski, K. Chwastek, I. Biondić, K. Miličević, Acta Phys. Pol. A 131, 1228 (2017)

47. A. J. Bergqvist, Physica B 233, 342 (1997)

48. F. Henrotte, A. Nicolet, K. Hameyer, COMPEL 25, 71 (2006)

49. V. François-Lavet, F. Henrotte, L. Stainer, L. Noels, C. Geuzaine, J. Comput. Appl. Math. 246, 243 (2013)

50. E. R. Madelung, Ann. Phys. 17, 861 (1905)

51. S. E. Zirka, Yu. I. Moroz, IEEE Trans. Magn. 31, 3509 (1995)

52. S. E. Zirka, Yu. I. Moroz, IEEE Trans. Magn. 35, 2090 (1999)

53. A. Cohen, Rheol. Acta 30, 270 (1991)

54. R. Jedynak, Rheol. Acta 54, 29 (2015) 